\title{
Contrast Volume/Raw eGFR Ratio for Predicting Contrast-Induced Acute Kidney Injury in Patients Undergoing Percutaneous Coronary Intervention for Myocardial Infarction
}

\author{
Hoon Suk Park ${ }^{a}$ Chan Joon Kim ${ }^{b}$ Jeong-Eun $\mathrm{Yi}^{\mathrm{b}}$ Byung-Hee Hwang ${ }^{\mathrm{b}}$ \\ Tae-Hoon Kim ${ }^{b}$ Yoon Seok Kohb Hun-Jun Park ${ }^{b}$ Sung-Ho Her ${ }^{b}$ \\ Sung Won Jang ${ }^{b}$ Chul-Soo Park ${ }^{b}$ Jong Min Lee ${ }^{b}$ Hee Yeol Kim ${ }^{b}$ \\ Doo Soo Jeon ${ }^{b}$ Pum-Joon Kim ${ }^{b}$ Ki-Dong Yoo ${ }^{b}$ Kiyuk Chang ${ }^{b}$ \\ Dong Chan Jin ${ }^{a} \quad$ Ki-Bae Seung ${ }^{b}$
}

Divisions of a Nephrology and ${ }^{b}$ Cardiology, Department of Internal Medicine, College of Medicine, The Catholic University of Korea, Seoul, Republic of Korea

\section{Key Words}

Estimated glomerular filtration rate $\cdot$ Body surface area normalization $\cdot$ Contrast-induced acute kidney injury

\begin{abstract}
Background: Considering that contrast medium is excreted through the whole kidney in a similar manner to drug excretion, the use of raw estimated glomerular filtration rate (eGFR) rather than body surface area (BSA)-normalized eGFR is thought to be more appropriate for evaluating the risk of contrast-induced acute kidney injury (CI-AKI). Methods: This study included 2,189 myocardial infarction patients treated with percutaneous coronary intervention. Logistic regression analysis was performed to identify the independent risk factors. We used receiver-operating characteristic (ROC) curves to compare the ratios of contrast volume (CV) to eGFR with and without BSA normalization in predicting CI-AKI. Results: The area under the curve (AUC) of the ROC curve for the model including all the significant variables such as diabetes mellitus, left ventricular ejection fraction, preprocedural glucose, and the CV/raw mod-
\end{abstract}

Hoon Suk Park and Chan Joon Kim contributed equally to this work. 
ification of diet in renal disease (MDRD) eGFR ratio was 0.768 [95\% confidence interval (CI), $0.720-0.816 ; p<0.001]$. When the CV/raw MDRD eGFR ratio was used as a single risk value, the AUC of the ROC curve was 0.650 (95\% CI, 0.590-0.711; $p<0.001$ ). When the CV/MDRD eGFR ratio with BSA normalization ratio was used, the AUC of the ROC curve further decreased to 0.635 (95\% CI, 0.574-0.696; $p<0.001$ ). The difference between the two AUCs was significant $(p=0.002)$. Conclusions: Raw eGFR is a better predictor for CI-AKI than BSA-normalized eGFR.

(C) 2015 S. Karger AG, Basel

\section{Introduction}

Recent studies have demonstrated that the ratio of contrast volume (CV) to either estimated glomerular filtration rate (eGFR) or creatinine clearance (eClCr) can be used as a single risk predictor for contrast-induced acute kidney injury (CI-AKI), since this ratio pharmacokinetically means the area under the curve (AUC) representing the systemic exposure of a contrast agent to the body $[1,2]$. When dealing with pharmacokinetic issues, it is more appropriate to use the raw eGFR in absolute numbers $(\mathrm{ml} / \mathrm{min})$ individualized with the removal of body surface area (BSA) normalization. However, some studies have taken the CV/BSAnormalized eGFR $\left(\mathrm{ml} / \mathrm{min} / 1.73 \mathrm{~m}^{2}\right)$ ratio for predicting CI-AKI, whereas others have applied eClCr ( $\mathrm{ml} / \mathrm{min}$ ) using the Cockcroft-Gault (CG) formula [3-6]. When drug dosing is adjusted in patients with chronic kidney disease, it is recommended to use eClCr derived from the CG formula or eGFR without BSA normalization $[7,8]$.

We hypothesized that raw eGFR is more appropriate for predicting CI-AKI than BSAnormalized eGFR and conducted this observational cohort study.

\section{Methods}

\section{Subjects}

Data of the study population was extracted from the Catholic Medical Center Percutaneous Coronary Intervention (COACT) Registry, a prospective multicenter, all-comer-based registry of patients undergoing percutaneous coronary intervention (PCI) with drug-eluting stents between January 1, 2004 and December 31, 2009 in eight hospitals affiliated with the Catholic Medical Center in South Korea. Among the 4,807 patients with follow-up laboratory data more than 2 weeks after index PCI, patients with end-stage renal disease and those on hemodialysis or peritoneal dialysis $(n=138)$ as well as renal transplant recipients $(n=$ 36) were excluded. A total of 4,633 patients were identified, and 2,189 myocardial infarction (MI) patients were included in our analysis.

All the patients received preprocedure intravenous hydration with half-normal saline at the rate of $1 \mathrm{ml} / \mathrm{kg}$ body weight (BW) per hour once MI was diagnosed. The contrast dose was left to the discretion of the interventional cardiologist. After exposure to contrast medium, the patients again received half-normal saline intravenously at a rate of $1 \mathrm{ml} / \mathrm{kg} / \mathrm{h}$ for $12 \mathrm{~h}$. This hydration rate was reduced in patients with decreased left ventricular ejection fraction (LVEF) or overt heart failure. The contrast agent used for primary PCI was iodixanol, an isosmolar nonionic dimer (Visipaque; GE Healthcare, Princeton, N.J., USA). The registry was approved by the Institutional Review Board of the Catholic Medical Center of South Korea (IRB No. XC11RIMI0107K).

Definition

CI-AKI was defined as an absolute increase of serum creatinine ( $\mathrm{SCr}$ ) by $>0.3 \mathrm{mg} / \mathrm{dl}$ or a relative increase of $\mathrm{SCr}$ by $>50 \%$, in accordance with the Acute Kidney Injury Network criteria. CI-AKI was diagnosed based on pre- and post-PCI SCr measurements. The pre-PCI SCr level was defined as that measured just before the procedure. The post-PCI SCr level was defined as the highest level measured within 7 days of PCI. BSA was calculated using the following Mosteller formula: 


$$
\operatorname{BSA}\left(\mathrm{m}^{2}\right)=\sqrt{\frac{\text { height }(\mathrm{cm}) \times \text { weight }(\mathrm{kg})}{3,600}} .
$$

Raw eGFR was calculated using the following equation:

$$
\text { Raw } \mathrm{eGFR}=\mathrm{eGFR} \text { with BSA normalization } \times \frac{\mathrm{BSA}}{1.73} .
$$

Modification of diet in renal disease (MDRD) eGFR $\left(\mathrm{ml} / \mathrm{min} / 1.73 \mathrm{~m}^{2}\right)$ was calculated using the following equation: $\mathrm{eGFR}=175 \times \mathrm{SCr}(\mathrm{mg} / \mathrm{dl})^{-1.154} \times(\text { age })^{-0.203} \times(0.742$ if female $)$. Underweight and overweight were defined as having a body mass index (BMI) $<18.5$ and $>25.0$, respectively.

\section{Collection of Clinical and Laboratory Data}

Variables included in the analysis were demographics (age, gender, height, and BW), medical history [presence of diabetes mellitus (DM), hypertension, and previous cerebrovascular accident (CVA)], smoking status, LVEF by echocardiography, laboratory findings (lipid profile, hemoglobin, preprocedural plasma glucose level measured in the emergency department before PCI, and pre- and post-PCI SCr levels measured during hospitalization), and CV used during PCI.

\section{Statistical Analyses}

Continuous variables are presented as means $\pm \mathrm{SD}$ and were compared using the $t$ test for independent samples. Variables that were not normally distributed are presented as medians and interquartile ranges and were compared with the Mann-Whitney U test. Categorical variables were compared using the $\chi^{2}$ test or Fisher's exact test, as appropriate. Binary logistic regression for estimating the hazard ratio and $95 \%$ confidence interval (CI) was used to identify the predictive factors for the development of CI-AKI. The variables with a variance inflation factor $>10$ were considered to have a multicollinearity problem. Analyses of receiveroperating characteristic (ROC) curves were conducted for risk variables for CI-AKI. ROC curves were then compared to determine which one was better for predicting CI-AKI. All $p$ values were two-tailed, and $\mathrm{p}$ values $<0.05$ were considered significant. Statistical analyses were performed using SPSS version 15.0 for Windows (SPSS, Chicago, Ill., USA) and the MedCalc version 11.6.1.0 statistical package (Med-Calc Software, Mariakerke, Belgium).

\section{Results}

\section{Baseline Demographic, Clinical, and Laboratory Profiles}

Of 2,189 patients, 157 (7.2\%) developed CI-AKI according to our definition. The patients in the CI-AKI group were older, and there were more female patients in this group. Height and BW were lower in the CI-AKI group. The CI-AKI group had a higher prevalence of DM, hypertension, and CVA. LVEF and hemoglobin were lower, while preprocedural plasma glucose levels were higher in the CI-AKI group than in the non-CI-AKI group. A comparison of lipid profiles between the two groups showed that both high-density lipoprotein cholesterol (HDL-C) and non-HDL-C levels were lower in the CI-AKI group. The CV and CV/BW were not significantly different between two groups. Preprocedural MDRD eGFR and raw eGFR (MDRD eGFR without BSA normalization) were lower in the CI-AKI group than in the non-CI-AKI group, with the difference in raw eGFR being larger. The CV/MDRD eGFR ratio was significantly larger in the CI-AKI group, and the CV/raw MDRD eGFR ratio was also larger compared to that in the non-CI-AKI group (table 1).

\section{Predictors of Developing CI-AKI}

Univariate and multivariate logistic regression analyses were performed to assess the effects of variables on CI-AKI development. Potential confounders (features that differed between the two groups) were included. Variables with a $\mathrm{p}$ value $<0.1$ in the univariate analyses were included in the multivariate model. No variables, including the CV/BW and CV/ 
Table 1. Baseline characteristics of the groups with and without CI-AKI

\begin{tabular}{|c|c|c|c|c|}
\hline & $\begin{array}{l}\text { Total } \\
(n=2189)\end{array}$ & $\begin{array}{l}\text { CI-AKI group } \\
(n=157)\end{array}$ & $\begin{array}{l}\text { Non-CI-AKI group } \\
(\mathrm{n}=2,032)\end{array}$ & $\mathrm{p}$ value \\
\hline Age, years & $62(53-71)$ & $68(57-74)$ & $62(52-71)$ & $<0.001^{\dagger}$ \\
\hline Female gender & $928(42.4)$ & $83(52.9)$ & $845(41.6)$ & 0.006 \\
\hline Height, $\mathrm{cm}$ & $165.0(159.0-170.0)$ & $163.0(155.0-168.0)$ & $165.0(159.0-170.0)$ & $0.001^{\dagger}$ \\
\hline $\mathrm{BW}, \mathrm{kg}$ & $65.0(58.0-73.0)$ & $63.0(55.0-68.5)$ & $65.0(58.0-73.0)$ & $<0.001^{\dagger}$ \\
\hline $\mathrm{BSA}, \mathrm{m}^{2}$ & $1.72 \pm 0.18$ & $1.67 \pm 0.18$ & $1.73 \pm 0.18$ & $<0.001$ \\
\hline $\mathrm{DM}$ & $719(32.8)$ & $91(58.0)$ & $628(30.9)$ & $<0.001$ \\
\hline Hypertension & $1,069(48.8)$ & $101(64.3)$ & $968(47.6)$ & $<0.001$ \\
\hline Smoking & $940(42.9)$ & $57(36.3)$ & $883(43.5)$ & 0.08 \\
\hline History of CVA & $143(6.5)$ & $22(14.0)$ & $121(6.0)$ & $<0.001$ \\
\hline LVEF, \% & $55.0(46.6-60.0)$ & $49.0(40.0-55.0)$ & $55.0(47.0-60.4)$ & $<0.001^{\dagger}$ \\
\hline Hemoglobin, g/dl & $13.9(12.6-15.3)$ & $13.0(11.0-14.4)$ & $14.0(12.7-15.3)$ & $<0.001^{\dagger}$ \\
\hline \multicolumn{5}{|l|}{ Preprocedural } \\
\hline plasma glucose, mg/dl & $136.0(112.0-186.0)$ & $171.0(124.5-239.8)$ & $135.7(112.0-182.0)$ & $<0.001^{\dagger}$ \\
\hline HDL-C, mg/dl & $40.0(34.0-46.0)$ & $38.0(32.0-45.0)$ & $40.0(34.0-46.0)$ & $0.013^{\dagger}$ \\
\hline Non-HDL-C, mg/dl & $136.0(111.0-162.0)$ & $130.0(103.0-153.0)$ & $136.0(111.0-163.0)$ & $0.019^{\dagger}$ \\
\hline $\mathrm{CV}, \mathrm{ml}$ & $250.0(200.0-350.0)$ & $250.0(190.0-384.8)$ & $250.0(200.0-350.0)$ & $0.831^{\dagger}$ \\
\hline $\mathrm{CV} / \mathrm{BW}, \mathrm{ml} / \mathrm{kg}$ & $4.0(2.8-5.5)$ & $4.3(2.8-6.1)$ & $3.9(2.8-5.5)$ & $0.091^{\dagger}$ \\
\hline \multicolumn{5}{|l|}{ Preprocedural MDRD } \\
\hline $\mathrm{eGFR}, \mathrm{ml} / \mathrm{min} / 1.73 \mathrm{~m}^{2}$ & $70.6(58.4-83.4)$ & $57.3(35.1-74.1)$ & $71.3(59.5-84.0)$ & $<0.001^{\dagger}$ \\
\hline \multicolumn{5}{|l|}{ Preprocedural raw } \\
\hline MDRD eGFR, $\mathrm{ml} / \mathrm{min}$ & $70.6(56.8-85.1)$ & $54.9(32.8-71.7)$ & $71.6(58.3-86.2)$ & $<0.001^{\dagger}$ \\
\hline CV/MDRD eGFR & $3.64(2.56-5.34)$ & $4.65(2.85-8.30)$ & $3.58(2.55-5.23)$ & $<0.001^{\dagger}$ \\
\hline CV/raw MDRD eGFR & $3.67(2.56-5.40)$ & $4.99(3.04-9.24)$ & $3.61(2.54-5.22)$ & $<0.001^{\dagger}$ \\
\hline
\end{tabular}

Values are presented as numbers with percentages in parentheses, means \pm SD, or medians with interquartile ranges in parentheses, as appropriate. ${ }^{\dagger}$ Calculated using the nonparametric Mann-Whitney U test.

raw MDRD eGFR ratios, were found to have multicollinearity. In the multivariate analysis, DM, LVEF, preprocedural plasma glucose, and the CV/raw MDRD eGFR ratio were found to be the independent risk factors for development of CI-AKI (table 2).

\section{Comparison of ROC Curves}

The Hosmer-Lemeshow test of the established model based on DM, LVEF, preprocedural glucose, and the CV/raw MDRD eGFR ratio showed a satisfactory goodness of fit ( $p=0.989$ ). The AUC of the ROC curve for this model including all the significant variables was 0.768 (95\% CI, $0.720-0.816$; $\mathrm{p}<0.001$ ), and the ROC curve exhibited $56.5 \%$ sensitivity and $85.3 \%$ specificity at a cutoff level of 0.0901 .

When the CV/raw MDRD eGFR ratio was used as a single risk value, the AUC of the ROC curve decreased to 0.650 (95\% CI, 0.590-0.711; $p<0.001$ ), which was a significant difference $(\mathrm{p}=0.001$ ) compared with the full-scale model. When the CV/MDRD eGFR with BSA normalization ratio was used, the AUC further decreased to 0.635 (95\% CI, 0.574-0.696; $\mathrm{p}<0.001$ ). The difference between the two AUCs for the ratios of CV/raw MDRD eGFR and the CV/MDRD eGFR with BSA normalization ratios was significant ( $p=0.002$; fig. 1 ).

Since the differences between the eGFRs calculated with and without BSA normalization are more marked in underweight or overweight persons than in persons within the normal range [9], we extracted 911 underweight and overweight patients from the whole study population. In this population, the AUC of the ROC curve of the CV/raw MDRD eGFR ratio for 
Table 2. Univariate and multivariate logistic regression model analyses of CI-AKI

\begin{tabular}{|c|c|c|c|c|}
\hline \multirow[t]{2}{*}{ Variables } & \multicolumn{2}{|l|}{ Univariate analysis } & \multicolumn{2}{|l|}{ Multivariate analysis } \\
\hline & HR $(95 \%$ CI) & $\mathrm{p}$ value & HR $(95 \%$ CI) & $\mathrm{p}$ value \\
\hline Age & $1.025(1.011-1.039)$ & $<0.001$ & $0.994(0.973-1.016)$ & 0.617 \\
\hline Female gender & $1.576(1.138-2.182)$ & 0.006 & $0.895(0.553-1.447)$ & 0.651 \\
\hline BW & $0.971(0.956-0.987)$ & $<0.001$ & $0.977(0.953-1.001)$ & 0.063 \\
\hline $\mathrm{DM}$ & $3.083(2.215-4.290)$ & $<0.001$ & $2.376(1.459-3.868)$ & 0.001 \\
\hline Hypertension & $1.982(1.414-2.780)$ & $<0.001$ & $1.269(0.810-1.989)$ & 0.299 \\
\hline Smoking & $0.742(0.530-1.039)$ & 0.082 & $0.966(0.610-1.529)$ & 0.883 \\
\hline History of CVA & $2.574(1.582-4.187)$ & $<0.001$ & $1.738(0.911-3.314)$ & 0.093 \\
\hline LVEF & $0.951(0.937-0.966)$ & $<0.001$ & $0.963(0.945-0.981)$ & $<0.001$ \\
\hline Hemoglobin & $0.768(0.712-0.828)$ & $<0.001$ & $0.906(0.807-1.017)$ & 0.093 \\
\hline Preprocedural plasma glucose & $1.005(1.004-1.007)$ & $<0.001$ & $1.003(1.001-1.005)$ & 0.015 \\
\hline HDL-C & $0.976(0.960-0.993)$ & 0.005 & $0.988(0.968-1.008)$ & 0.222 \\
\hline Non-HDL-C & $0.995(0.990-0.999)$ & 0.013 & $1.000(0.995-1.005)$ & 0.988 \\
\hline $\mathrm{CV} / \mathrm{BW}$ & $1.077(1.002-1.158)$ & 0.045 & $0.946(0.840-1.066)$ & 0.365 \\
\hline CV/raw MDRD eGFR & $1.115(1.077-1.155)$ & $<0.001$ & $1.073(1.019-1.131)$ & 0.008 \\
\hline
\end{tabular}

HR = Hazard ratio

Fig. 1. Comparison of the ROC curves of the model including all the significant variables as well as the CV/raw MDRD eGFR and the CV/MDRD eGFR ratios.

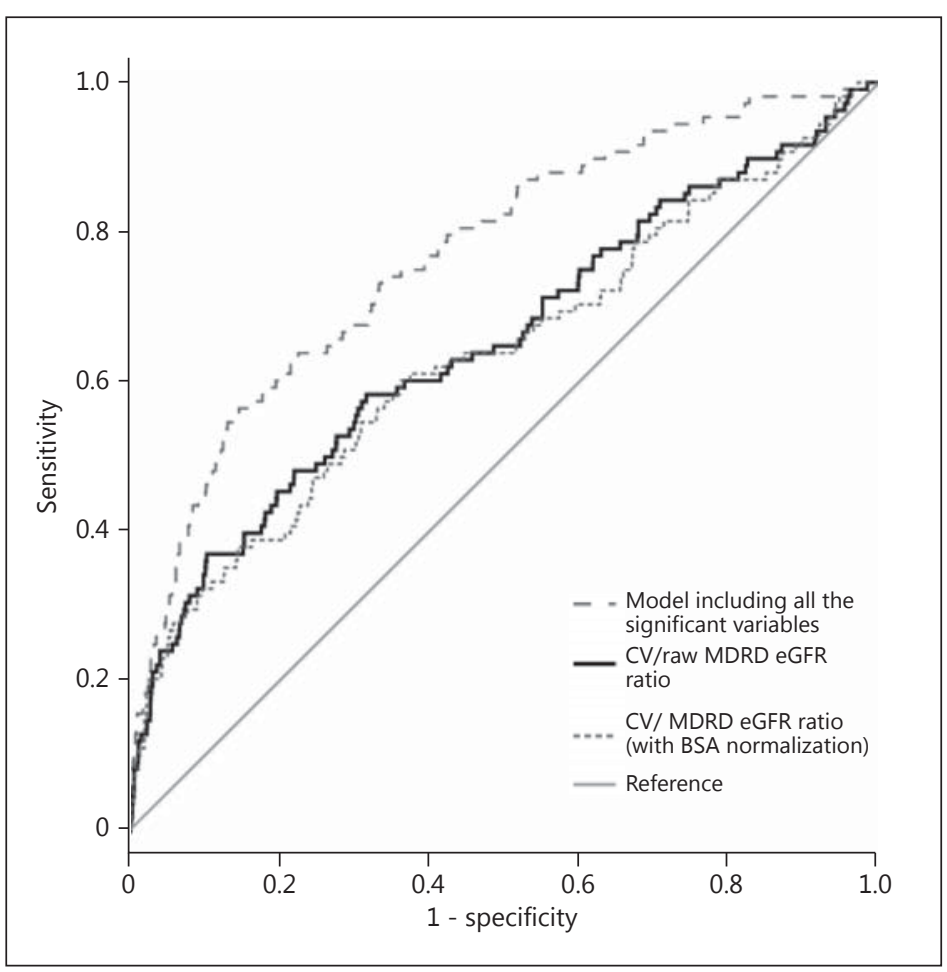

predicting CI-AKI was 0.655 (95\% CI, 0.566-0.744; $\mathrm{p}<0.001$ ), which is almost the same value compared with that of the whole population, whereas the AUC of the ROC curve of the CV/MDRD eGFR with BSA normalization ratio was 0.628 (95\% CI, 0.535-0.72; $p=0.003$ ), which is lower than that of the whole population. The difference between the two AUCs was significant ( $p=0.006$; fig. 2 ). 


\section{CardioRenal Medicine}

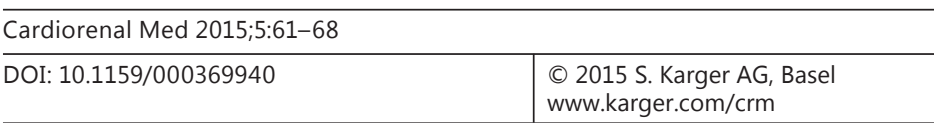

Park et al.: CV/Raw eGFR Ratio for Predicting CI-AKI in Patients Undergoing PCI for MI

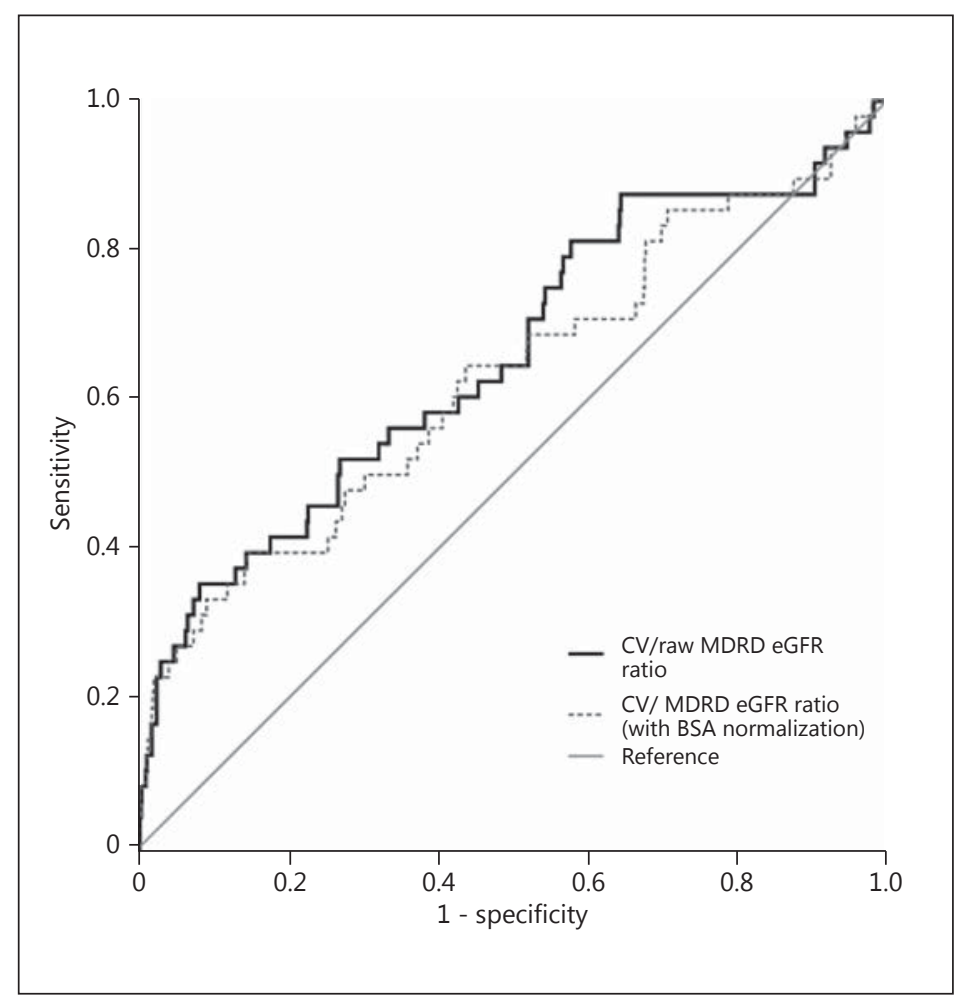

Fig. 2. Comparison of the ROC curves of the $\mathrm{CV} /$ raw MDRD eGFR and the CV/MDRD eGFR ratios of the underweight or overweight patients only, extracted from the whole study population. 
Recent studies investigating the predictive value of the $\mathrm{CV} / \mathrm{eClCr}$ or $\mathrm{CV} / \mathrm{eGFR}$ ratios for CI-AKI suggested that maximally acceptable CV should be individually calculated by multiplying the cutoff value of the ratio and each patient's eGFR $[1,4-6]$. The use of eGFR with BSA normalization can lead to an underestimation of the actual contrast removal rate in overweighed individuals and to an overestimation in underweighted ones [12], so the use of raw eGFR without BSA normalization rather than BSA-normalized eGFR is more appropriate when dealing with the toxicity of contrast medium. It is possible that patients with a higher BMI have higher actual or raw GFRs and are at a lower risk for the development of CI-AKI. Balemans et al. [13] reported that a low BMI was significantly associated with the development of CI-AKI. Clinicians need to be aware of the implication of individualized eGFR based on patient-specific BSA.

Cigarroa et al. [14] showed that a larger volume of contrast medium relative to BW is likely to contribute to the development of CI-AKI to determine the maximal acceptable contrast dose (MACD). The MACD is calculated by $5 \mathrm{ml}$ of contrast $\times \mathrm{BW}(\mathrm{kg}$ ) (maximum, $300 \mathrm{ml}$ )/baseline $\mathrm{SCr}(\mathrm{mg} / \mathrm{dl})$, an empiric equation that they had utilized over the past 10 years. Although the MACD has been validated in several studies, it is infrequently used in clinical practice because in a significant number of cases, CI-AKI can develop even if the MACD is not exceeded $[15,16]$. The $\mathrm{CV} /$ raw MDRD eGFR ratio pharmacokinetically means the AUC representing the systemic exposure of the contrast agent, so it can be a better alternative.

So far, various studies have demonstrated that the $\mathrm{CV} / \mathrm{eClCr}$ or $\mathrm{CV} / \mathrm{eGFR}$ ratio is a significant risk factor for CI-AKI in patients undergoing PCI. Some included all patients with ischemic heart disease (IHD) while others included a selective group such as ST-segment elevation MI (STEMI). A recent article retrospectively analyzed patients with stable angina pectoris (AP), unstable AP (UAP), non-STEMI (NSTEMI) and STEMI [17]. The study population was divided by $\mathrm{CV} / \mathrm{eGFR}$ ratio ranges of $<2.0,2.0-2.9$, and $\geq 3.0$, based on the suggestion in the former study by Gurm et al. [18] that the restriction of CV to less than thrice and preferably twice of the eGFR might prevent $\mathrm{CI}-\mathrm{AKI}$. The study showed that a CV/eGFR ratio $\geq 3.0$ was a significant risk factor of CI-AKI in the stable AP group, but not in the UAP, NSTEMI and STEMI groups. However, Gurm et al. [18] also retrospectively analyzed the whole patient group with all kinds of IHD undergoing PCI. Therefore, the suggestion by Gurm et al. [18] of minimizing CV may be generally acceptable for the patients with IHD for PCI, but it cannot be applied to relatively hemodynamically unstable patients. We may need another range of CV/ eGFR ratios for using contrast media safely in patients with UAP and MI. In contrast, other prospective studies showed that the $\mathrm{CV} / \mathrm{eGFR}$ ratio was a significant predictor for CI-AKI in the patients with STEMI $[4,6]$. A large and prospective trial is required for the further confirmation that the $\mathrm{CV} / \mathrm{eGFR}$ ratio is a significant variable in hemodynamically unstable patients such as those with MI.

Our study has some limitations. The cases in which an intra-aortic balloon pump was used due to severe hemodynamic instability could not be identified, so that some patients might develop AKI due to hemodynamic reasons, although CI-AKI is multifactorial, or a complex syndrome of AKI after the administration of contrast media.

In conclusion, our study showed that as a single risk value, the CV/raw MDRD eGFR ratio predicted CI-AKI better than the CV/BSA-normalized MDRD eGFR ratio; this strongly supports the idea that raw GFR without BSA normalization, in the unit of $\mathrm{ml} / \mathrm{min}$ rather than $\mathrm{ml} / \mathrm{min} / 1.73 \mathrm{~m}^{2}$, is considered when predicting CI-AKI.

\section{Disclosure Statement}

The authors have no conflicts of interest to disclose. 


\section{CardioRenal Medicine}

\section{References}

1 Laskey WK, Jenkins C, Selzer F, Marroquin OC, Wilensky RL, Glaser R, Cohen HA, Holmes DR Jr: Volume-tocreatinine clearance ratio: a pharmacokinetically based risk factor for prediction of early creatinine increase after percutaneous coronary intervention. J Am Coll Cardiol 2007;50:584-590.

-2 Nyman U, Bjork J, Aspelin P, Marenzi G: Contrast medium dose-to-GFR ratio: a measure of systemic exposure to predict contrast-induced nephropathy after percutaneous coronary intervention. Acta Radiol 2008;49: 658-667.

-3 Yoon HJ, Hur SH: Determination of safe contrast media dosage to estimated glomerular filtration rate ratios to avoid contrast-induced nephropathy after elective percutaneous coronary intervention. Korean Circ J 2011; 41:265-271.

4 Liu Y, Tan N, Zhou YL, He PC, Luo JF, Chen JY: The contrast medium volume to estimated glomerular filtration rate ratio as a predictor of contrast-induced nephropathy after primary percutaneous coronary intervention. Int Urol Nephrol 2012;44:221-229.

5 Tan N, Liu Y, Zhou YL, He PC, Yang JQ, Luo JF, Chen JY: Contrast medium volume to creatinine clearance ratio: a predictor of contrast-induced nephropathy in the first 72 hours following percutaneous coronary intervention. Catheter Cardiovasc Interv 2012;79:70-75.

6 Mager A, Vaknin Assa H, Lev EI, Bental T, Assali A, Kornowski R: The ratio of contrast volume to glomerular filtration rate predicts outcomes after percutaneous coronary intervention for ST-segment elevation acute myocardial infarction. Catheter Cardiovasc Interv 2011;78:198-201.

7 Hudson JQ, Nyman HA: Use of estimated glomerular filtration rate for drug dosing in the chronic kidney disease patient. Curr Opin Nephrol Hypertens 2011;20:482-491.

-8 Jones GR: Estimating renal function for drug dosing decisions. Clin Biochem Rev 2011;32:81-88.

9 Choi JY, Nam SA, Jin DC, Kim J, Cha JH: Expression and cellular localization of inducible nitric oxide synthase in lipopolysaccharide-treated rat kidneys. J Histochem Cytochem 2012;60:301-315.

10 Jones GR: Estimated GFR for drug dosing: a bedside formula. Am J Kidney Dis 2009;54:982-983, author reply 985-986.

11 Stevens LA, Nolin TD, Richardson MM, Feldman HI, Lewis JB, Rodby R, Townsend R, Okparavero A, Zhang YL, Schmid $\mathrm{CH}$, Levey AS: Comparison of drug dosing recommendations based on measured GFR and kidney function estimating equations. Am J Kidney Dis 2009;54:33-42.

-12 Nyman U, Almen T, Aspelin P, Hellstrom M, Kristiansson M, Sterner G: Contrast-medium-Induced nephropathy correlated to the ratio between dose in gram iodine and estimated GFR in ml/min. Acta Radiol 2005;46:830842.

13 Balemans CE, Reichert LJ, van Schelven BI, van den Brand JA, Wetzels JF: Epidemiology of contrast materialinduced nephropathy in the era of hydration. Radiology 2012;263:706-713.

14 Cigarroa RG, Lange RA, Williams RH, Hillis LD: Dosing of contrast material to prevent contrast nephropathy in patients with renal disease. Am J Med 1989;86:649-652.

15 Brown JR, Robb JF, Block CA, Schoolwerth AC, Kaplan AV, O'Connor GT, Solomon RJ, Malenka DJ: Does safe dosing of iodinated contrast prevent contrast-induced acute kidney injury? Circ Cardiovasc Interv 2010;3: 346-350.

16 Freeman RV, O’Donnell M, Share D, Meengs WL, Kline-Rogers E, Clark VL, DeFranco AC, Eagle KA, McGinnity JG, Patel K, Maxwell-Eward A, Bondie D, Moscucci M: Nephropathy requiring dialysis after percutaneous coronary intervention and the critical role of an adjusted contrast dose. Am J Cardiol 2002;90:1068-1073.

17 Abe D, Sato A, Hoshi T, Kakefuda Y, Watabe H, Ojima E, Hiraya D, Harunari T, Takeyasu N, Aonuma K: Clinical predictors of contrast-induced acute kidney injury in patients undergoing emergency versus elective percutaneous coronary intervention. Circ J 2014;78:85-91.

18 Gurm HS, Dixon SR, Smith DE, Share D, Lalonde T, Greenbaum A, Moscucci M: Renal function-based contrast dosing to define safe limits of radiographic contrast media in patients undergoing percutaneous coronary interventions. J Am Coll Cardiol 2011;58:907-914. 